Official Publication of the International Association for Cognitive

Education and Psychology

www.iacep-coged.org

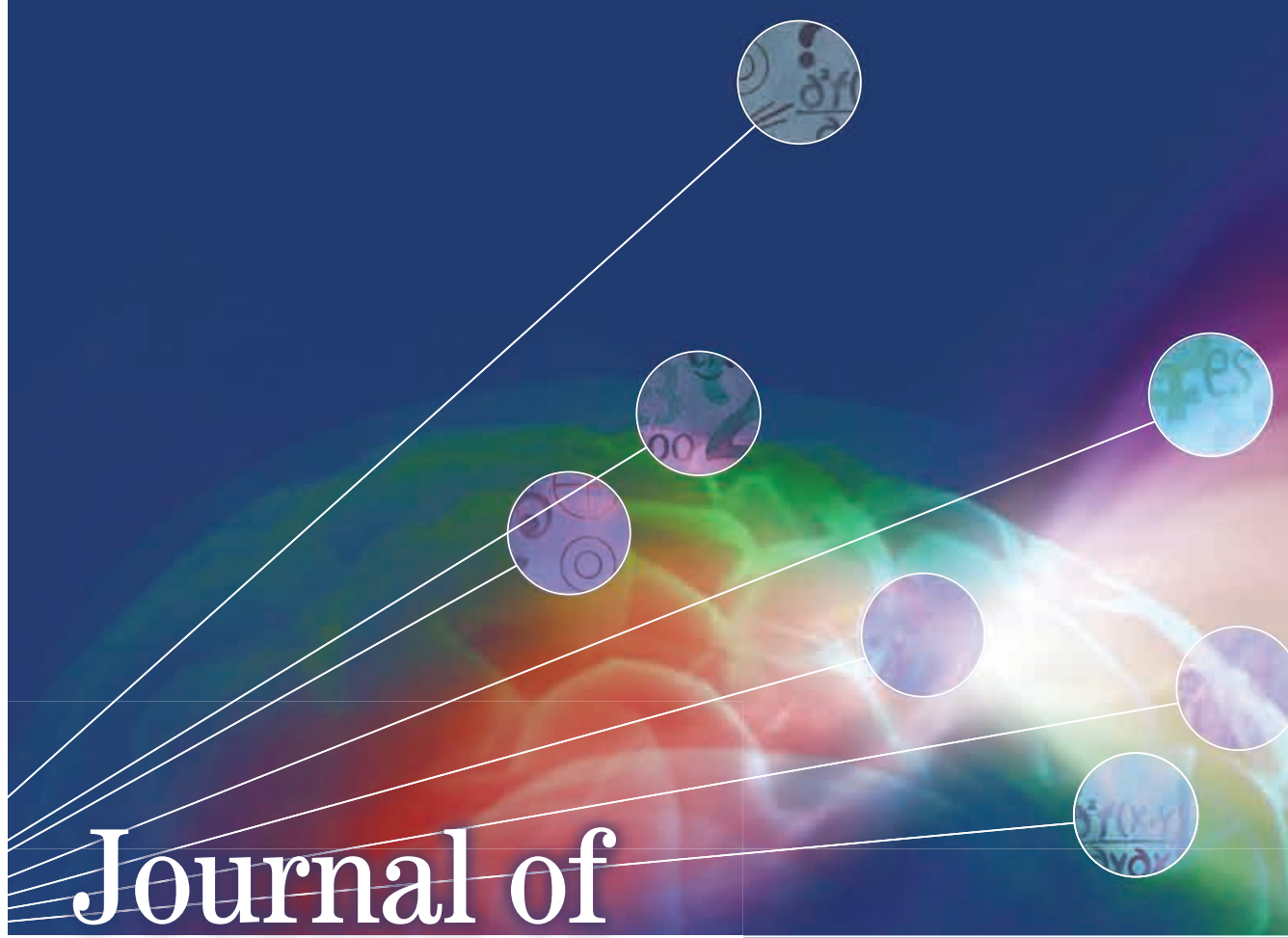

Cognitive Education and Psychology

With the Compliments of Springer Publishing Company, LLC 
Journal of Cognitive Education and Psychology

Volume 10, Number 1, 2011

\title{
Three Lines in the Emergence of Prelinguistic Communication and Social Cognition
}

\author{
Ulf Liszkowski \\ Max Planck Research Group Communication Before Language, \\ Max Planck Institute for Psycholinguistics, and \\ Donders Institute for Brain, Cognition, and Behaviour, \\ Radboud University, Nijmegen, The Netherlands
}

\begin{abstract}
Sociocultural theories of development posit that higher cognitive functions emerge through socially mediated processes, in particular through language. However, theories of human communication posit that language itself is based on higher social cognitive skills and cooperative motivations. Prelinguistic communication is a test case to this puzzle. In the current review, I first present recent and new findings of a research program on prelinguistic infants' communication skills. This research provides empirical evidence for a rich social cognitive and motivational basis of human communication before language. Next, I discuss the emergence of these foundational skills. By considering all three lines of development, and by drawing on new findings from phylogenetic and cross-cultural comparisons, this article discusses the possibility that the cognitive foundations of prelinguistic communication are, in turn, mediated by social interactional input and shared experiences.
\end{abstract}

Keywords: cross-cultural; socio-historic; infant communication; pointing; gesture

$\mathrm{H}$ uman cognition is unique and diverse in many ways. Interestingly, the complexities of human cognition emerged in such an evolutionarily short time span that they cannot easily be accounted for by natural evolution alone (e.g., Richerson \& Boyd, 2005). This has led to the argument and some intriguing evidence that the emergence of the complexities and unique aspects of human cognition can be reduced to the advent of a single foundational ability—namely, the understanding of others as intentional beings (Tomasello, 1999), together with a cooperative motivation to interact with others ("shared intentionality"; Tomasello, Carpenter, Call, Behne, \& Moll, 2005). The argument is that these foundational social cognitive and social motivational skills led to species-specific ways of interacting, cumulating in modern human linguistic exchanges. These forms of human interaction and communication then enabled a faster cultural evolution of complex human cognition over a shorter historic period. Specifically, 
the advent of language transformed natural lines of cognition inherited from nonhuman ancestors into uniquely human forms of perspectival and dialogical thinking.

Vygotsky $(1962,1978)$ and Luria (1976) argued, and provided some evidence to show, that cognitive skills inherited from a natural (evolutionary) line of development interacted with cultural tools inherited from a sociohistoric line of development. Together, these two lines mediated the individual (ontogenetic) line of development. The central claim of Vygotsky's sociocultural theory of development was that every higher cognitive function first emerges on an interpersonal plane and then, through cultural mediation and internalization, on an intrapersonal plane. In particular, Vygotsky argued that language as a cultural tool mediates the ontogenetic emergence of higher cognitive functions. In support of this view, more recent cross-linguistic research has demonstrated how language influences cognition in various domains such as space, perception, and numerical knowledge (e.g., Boroditsky, 2009; Frank, Everett, Fedorenko, \& Gibson, 2008; Haun et al., 2006; Levinson, 2003). Social origins' views of human cognition thus propose that very few, if any, of humans' unique cognitive skills are a product of individual processes alone. Instead, they suggest that human complex cognition is socially constructed in a dialectic manner from naturally inherited skills and sociohistorically developed cultural tools, such as symbols, artifacts, and, in particular, language.

Following social origins views (see Carpendale \& Lewis, 2004), social interaction and communication play a key role in the emergence of unique forms of human cognition. However, one might also argue that specific forms of cognition are the departure point to get interaction and communication off the ground (e.g., Knoblich \& Sebanz, 2008). How does human communication work, and how does language emerge in the first place? Unlike animal communication, which relies on either phylogenetically or ontogenetically ritualized action patterns and natural meaning (Tinbergen, 1951; Tomasello, 2008), human communication is an inferential process that requires sophisticated cognitive skills. Philosophical accounts define human communication by its intentionality and cooperative processes, not by language alone (Grice, 1957; Searle, 1969; Sperber \& Wilson, 1995). Searle emphasized that acts of reference are based on the utterer's intentions and are underlain by a limited set of distinct intentional "forces" or acts, such as declaring, requesting, and so forth. Grice showed that beyond the linguistic code, communication entails a special structure of embedded intentions (the intention that others understand one's intentions) and is based on cooperative principles by which interlocutors work together toward understanding each other. This special structure is foundational to human communication and makes it possible to infer meanings beyond explicitly conveyed language. Quine (1960) further showed that translations of linguistic codes are impossible and must be based on pragmatics and inferences, because the linguistic code alone underdetermines what people intend to communicate. Thus, to acquire a linguistic (or other arbitrary) code in the first place, one already needs foundational communication skills to understand what is being coded. The ontogenetic argument is thus that human communication already relies on higher cognitive skills that run much deeper than language alone and enable acquisition of linguistic codes in the first place (Bates, 1979; Bruner, 1981; Tomasello, 2008). The dialectic puzzle is then that language and communication, on the one hand, mediate higher cognitive functions, but, on the other hand, are mediated themselves by deeper cognitive skills. A test case to this puzzle is how infants communicate before language.

Following up on the ontogenetic argument, in the first part of this article I will review evidence on whether infants indeed communicate with deeper social cognitive skills and cooperative motives prior to language. Infants communicate with gestures before they speak 
(Bates, Camaioni, \& Volterra, 1975), but the central question is whether communication before language is already underpinned by deeper cognitive and motivational processes that enable language acquisition in the first place. I will present a series of recent and new studies that show that prelinguistic infants indeed communicate in cognitively and motivationally richer ways than previously thought. These findings thus demonstrate that foundational social cognitive skills are not mediated by language, but instead emerge prior to and thus independently of language.

The second part of this article raises the question how, in turn, these foundational social and cognitive skills of prelinguistic communication emerge, in particular, in the absence of cultural and symbolic tools such as language. Answers to this question are still far from conclusive, but I will lay out some questions and discuss emerging empirical findings from recent and new phylogenetic and cross-cultural comparisons. The general standpoint will be that we need not defect a priori to a nativist or "core systems" stance in explaining the foundations of prelinguistic communication and cognition. Instead, it will be a useful exercise to consider again all three lines of development—-the natural (evolutionary), the individual (ontogenetic), and the sociohistoric or cross-cultural lines-to investigate social processes in the ontogeny of prelinguistic communication and cognition.

\section{PRELINGUISTIC FOUNDATIONS OF HUMAN COMMUNICATION}

Before they speak, infants communicate with various kinds of gestures. Of interest to our question is the most natural type of gestures, one that does not rely on codified and socially transmitted meaning. These are deictic gestures, such as showing and pointing, which work through ways of presenting others with an aspect in the environment, in the hope that others then figure out the intended message. Deictic communication therefore does not rely on semantics or symbol use. The advantage is that one can use the same gesture (e.g., pointing) to express a myriad of different things simply by presenting others with relevant aspects of the environment on the background of a shared current situation. However, deictic communication is heavily dependent on a deeper social cognitive understanding of others' intentionality and shared situations. Specifically, deictic communication requires an understanding of others' referential, social, and communicative intentions, that is, respectively, what aspect someone indicates, why that aspect is indicated, and that the two previous questions are triggered because of the communicative act (Sperber \& Wilson, 1995; Tomasello, Carpenter, \& Liszkowski, 2007).

Previous research has suggested that infants' deictic communication, in particular, the pointing gesture, which emerges around 12 months of age (Leung \& Rheingold, 1981), initially does not involve any of these cognitive and motivational complexities. Instead, the respective social cognitive understanding emerges only later, through parental scaffolding, pretty much in a way that socioconstructivist accounts of development would suggest (e.g., Carpendale \& Lewis, 2004). These cognitively "lean" hypotheses of infant pointing posited that initially (a) infants point noncommunicatively (Bates et al., 1975; Desrochers, Morissette, \& Ricard, 1995); (b) pointing is nonreferential and does not involve a social cognitive understanding of the recipients' attention (Moore \& D'Entremont, 2001); and (c) infants' motivation is mainly egocentric, to obtain objects or attention to the self (Bates et al., 1975; Gomez, Sarria, \& Tamarit, 1993; Moore \& D'Entremont, 2001). Recently, my colleagues and I have tested these claims and shown in a series of studies that infant pointing instead already shares much, if not all, of the social and cognitive complexities of the adult version of this communicative act from the beginning. 


\section{Intention to Communicate}

Twelve-month-olds use the pointing gesture intentionally to communicate. Two main sources of evidence come from a paradigm in which interesting events elicit infant pointing. We manipulated the experimenter's reaction either after or before the infant pointed. For example, the experimenter did not react to infants' pointing. In that case, infants persisted in their communicative goal and augmented their signal by repeating their pointing and increasing their vocalizations significantly more than when the adult typically reacted by sharing attention and interest (Liszkowski, Carpenter, Henning, Striano, \& Tomasello, 2004). This kind of persistence is indicative of pursuing the intention to communicate with others. Even more clearly, before infants initiated a point, they considered whether a recipient attended to them and so could see their point. When an adult turned sideways and did not look at infants (and so could not possibly see the pointing gesture), infants pointed significantly less to an interesting event than when the adult was turned toward them and so could see and react to their visual gesture (Liszkowski, Albrecht, Carpenter, \& Tomasello, 2008). These findings thus establish experimentally that 12 -month-olds point with the intention to communicate.

It has been debated whether infants also point non-communicatively. Bates et al. (1975; Desrochers et al., 1995) claimed that infants' first points are noncommunicative and only develop into communicative pointing. But recently, Delgado, Gómez, and Sarriá (2009) showed that noncommunicative pointing does not cease but coexists with communicative pointing in older 3- to 6-year-old children. These authors suggest a dual function of pointing (i.e., communicative and private), analogously to a Vygotskian perspective on speech. However, in a recent study (Liszkowski \& Tomasello, in press), we found that only infants who already pointed with the index-finger pointed more frequently, accompanied their points more often with vocalizations, and comprehended pointing in terms of referential intentions, compared to other same-aged infants who sometimes indicated with the whole hand. Based on these correlational findings, the aforementioned experimental evidence, and the fact that indexfinger pointing is a late-emerging joint attention behavior that is preceded by other gestures such as offers, shows, gives, and requests (Carpenter, Nagell, \& Tomasello, 1998), it is reasonable to believe that pointing, at least index-finger pointing, starts out with a communicative function. It may, however, be occasionally observed in noncommunicative situations, first when a communicative partner is not available or responding, and later for other private (e.g., mnemonic) functions (Delgado et al., 2009).

\section{Referential Intentions}

There is by now good evidence that 12-month-olds also point with referential intentions to present others with a subject of their communication. For example, in the paradigm in which interesting events elicited infant pointing, we had an experimenter misidentify infants' referents and attend solely either to the infants' face or to an irrelevant object nearby the intended referent, both times emoting positively to the infant (Liszkowski et al., 2004; Liszkowski, Carpenter, \& Tomasello, 2007a). In both these cases of referential misunderstandings, infants attempted to redirect the recipient's attention by repeating their pointing to the intended referent significantly more often than when the recipient had correctly identified the referent. Further, they vocalized more and looked more often at the experimenter in cases of referential misunderstandings, providing further indications of persistence and surprise. 
In these studies, infants' pointing was not simply a response to the presence of interesting stimuli. We found in further studies that infants communicated about ceased events or objects that were not present at the time of testing. For example, when infants had attended to an interesting event and it had ceased, they then pointed to its previous, now-empty location, depending on how a recipient had reacted to it before (Liszkowski, Carpenter, \& Tomasello, 2007b). Further, in a request-paradigm, to obtain a desirable object that was absent from its usual location at the moment of request, infants pointed to the now-empty location to request the absent entity (Liszkowski, Schäfer, Carpenter, \& Tomasello, 2009). Communication beyond the immediate "here and now" has previously been taken to be a design feature of human language ("displacement"; Hockett, 1960). Our findings establish for the first time that already before language, infants point with the intention to refer others to specific events and entities, and sometimes even absent referents.

\section{Social Intentions}

Because communication is a collaborative activity in which the sender and recipient both work together by aligning and helping each other to achieve mutual understanding, one question is whether infants' communication, too, is motivated by these aspects. One main finding of our studies was that infants indeed point at interesting events to share their interest about these events with others. For example, when an adult only oriented to the infant's referent but then did not comment on it (Liszkowski et al., 2004), or when the adult commented about the referent in an unenthusiastic way and signaled that the adult's interest did not match the infant's interest (Liszkowski et al., 2007a), infants' intention was not satisfied as reflected in their differential pattern of pointing: When the experimenter did not comment at all, infants persisted and repeated their pointing; when the experimenter's comment was uninterested instead of positive, infants did not persist but ceased pointing over trials (although they remained generally interactive with the adult throughout the experiment). Crucially, when the adult already knew about the referent (Liszkowski et al., 2007b), infants still pointed but only if the adult had expressed interest in it, presumably to share and express their alignment with the adult's expression of attitude. These findings show that infants do not only want to share the visual focus on a referent; they want to express and share their attitudes about a referent, too. This type of expressive pointing is also used in referential proto-conversations between caregivers and infants, as indicated by a correlational use (Liszkowski \& Tomasello, in press), and instrumental in establishing common shared background knowledge (Liebal, Carpenter, \& Tomasello, 2010).

Another main finding of our studies was that infants also point to help others. We designed a new search paradigm in which infants were confronted with an adult searching for one of two things. Findings were that infants pointed more to the relevant than irrelevant object to help the adult find it, with no requestive accompaniments or particular interest in the object (Liszkowski, Carpenter, Striano, \& Tomasello, 2006), and they did this more so when the adult needed help to find it than when she did not (Liszkowski et al., 2008). More recently, we found that infants also provide information to warn others in anticipation of a negative action outcome (Knudsen \& Liszkowski, 2010). In the study, an adult experienced unforeseen negative effects (pain or disgust) when accidentally bumping into an object in the course of her play. Subsequently, she removed the object out of her way. In her absence, someone else accidentally pushed the object back into the way. When she reappeared, infants 
spontaneously pointed out the object, even though the experimenter did not search for anything and the object did not move in interesting ways. Infants pointed significantly less in control conditions, when the object was either positive (thus not evoking negative side effects) or the experimenter had witnessed the location change (and thus already knew about the problem). Informing others to help them find something and warning others to help them avoid something are both based on a prosocial motivation. Infants' informative pointing and warning may be the ontogenetically earliest evidence for altruistic helping without direct benefit for the self.

\section{Social Cognitive Skills of Infant Pointing}

The reported studies directly tested 12-month-olds' social cognitive understanding of others' intentional and epistemic states. In the event paradigm, infants understood others' attention as evidenced by significantly more pointing when the adult had not yet seen the event than when she already had. Infants' pointing further revealed an understanding of the adult's attitudinal relations to the referent (i.e., whether she was interested in it or not; Liszkowski et al., 2007a, 2007b). In the search paradigm, infants understood an adult's intention to find an object and they understood whether the adult was knowledgeable or ignorant (Liszkowski et al., 2006; 2008). The warning paradigm further clarified that infants anticipate others' actions based on an understanding of their goals and epistemic states, even in the absence of behavioral manifestations like effortful trying or search behaviors (Knudsen \& Liszkowski, 2010). The latter study suggests an emerging belief-desire psychology of action predictions in the service of collaborative communication (see Knudsen \& Liszkowski, 2011, for further evidence of 18- and 24-month-olds' productive usage of "theory-of-mind" skills in a paradigm of anticipatory correcting). Further studies on infants' comprehension of pointing show that 12-month-olds also comprehend others' pointing in terms of the pointer's underlying referential intentions (Gliga \& Csibra, 2009), and that 12-month-olds' referential understanding of pointing is bidirectional as evidenced by correlations of infants' production and comprehension of pointing to occluded referents (Behne, Liszkowski, Carpenter, \& Tomasello, 2011; Liszkowski \& Tomasello, in press).

In sum then, recent experimental findings provide a new look at infant pointing as a fully communicative act including full-fledged reference on a mental level, even to absent entities, with cooperative motives such as sharing and helping, all before language has emerged. These findings thus provide ontogenetic evidence that human communication is based on social cognitive and motivational skills that run much deeper than language alone, and are manifest in the gestural modality of deictic communication around 12 months of age.

\section{ORIGINS OF PRELINGUISTIC COMMUNICATION}

How do the core social cognitive and motivational skills of human communication emerge prior to and thus independently of language? Our knowledge in this respect is still scarce. If we think of these skills as a core cognitive processing system that is part of human cognition from the beginning (for other examples of core cognitive systems, see Spelke, 1994; Carey, 2009), one question is to what extent infants' prelinguistic communication skills are shared with nonhuman ancestors. Evidence for an evolutionary association would refute strong claims of social constructivism and suggest that infants' skills are inherited from the natural line of development. Another question is whether prelinguistic communication skills are in fact universal 
across different cultural settings. For example, if we found cross-cultural differences in their existence before language, this would make "core systems" accounts less likely and suggest an influence of sociocultural lines of development. From an individual line of development, the most central question to social origins accounts is whether infants' skills emerge through and are mediated by social-interactional input and communicative practices.

With regard to inherited skills from the evolutionary line, one thing to note is that nonhuman primates communicate in various sophisticated ways. Current research (see Call \& Tomasello, 2007, for an overview) shows that great apes' communication is most flexible in the gestural modality and clearly involves the intention to address others. However, the origins and repertoire of apes' gestures seem fundamentally different from those of humans. Most gestures are ontogenetically ritualized from individual action schemes. These gestures are thus not learned via social imitation but originate from individual chaining of abbreviated behaviors. As a consequence, quite a few of apes' gestures are idiosyncratic and not shared with other individuals. Social-cognitively, apes presumably do not understand their gestural communication from another individual's perspective, that is, bidirectionally (Tomasello, 2008). This has striking consequences for deictic acts of reference such as pointing. Apes point in captivity for humans (Leavens, Russell, \& Hopkins, 2005) but apparently not for one another, neither in the wild nor in zoos (Povinelli, Bering, \& Giambrone, 2003; Tomasello, 2006). Importantly, apes' pointing differs from that of prelinguistic human infants in at least two fundamental ways. First, apes only point to request things, usually food, but not to share interest and not to inform others helpfully (see Bullinger, Zimmermann, Kaminski, \& Tomasello, 2010). This difference on the level of social intentions may be one of the reasons why apes also do not understand a human's pointing gesture. For example, on object choice tasks in which a human indicates one of two buckets that contains a hidden food, apes follow the point to the bucket. But unlike 12-month-olds (Behne et al., submitted), apes do not understand why the human pointed to the bucket; that is, they presumably fail to understand the human's social intention to helpfully inform them and, therefore, do not locate the hidden referent (Tomasello, 2006). Second, apes' pointing is also different on the level of referential intentions. In a recent study (Liszkowski et al., 2009), apes requested a food item that was currently not visible (occluded) by approaching its location with an extended arm and/or index finger. However, unlike human 12-month-olds, they did not request a food item that was currently absent from its usual location by pointing to the empty but mutually known location. Presumably, apes do not use their pointing gestures within a shared common ground to communicate their referential intent, but to recruit help in obtaining something. Their usage of pointing is also not bidirectional, considering that they did request a concealed food item from a human but did not understand the human's informative point to a hidden reward. Apes' gestures, and in particular their pointing gestures, are thus fundamentally different from prelinguistic infants' pointing in terms of the underlying social and referential intentions and the bidirectional understanding of the communicative act.

With regard to the sociocultural line, anthropological and cross-linguistic observations may suggest that infants' prelinguistic pointing is not universal but a result of Western culture-specific practices (e.g., Wilkins, 2003). Indeed, several developmental studies have documented infants' pointing gestures in the context of book reading (e.g., Murphy, 1978), which is a very culture-specific activity. One suspicion is that it is only in Western cultures in which whole industries are devoted to producing numerous artifacts for infants, and in which caretakers spend a lot of time interacting, playing, and pointing for their infants, that infants 
point with their fingers at things before they begin to speak. This would run against the claim that pointing, and specifically index-finger pointing (Liszkowski \& Tomasello, in press), plays a pivotal role before language.

Studies to date have investigated infant pointing in North America, Europe, and Japan (see Butterworth, 2003), but no study to date has systematically compared infants' and caregivers' pointing in a standardized setting across cultures. In a recent study (Liszkowski, Brown, Callaghan, Takada, \& de Vos, 2010), we decorated a room with a set of different items, and caregivers and infants were invited to look at these together, a situation broadly analogous to an exhibit or a visit to a zoo (based on Liszkowski \& Tomasello, in press). We used the task with babies around their first birthdays in seven different countries in India, Indonesia, Papua New Guinea, Japan, Mexico, Peru, and Canada—cultures that were indigenous or rural and had independent or interdependent socialization goals. Main findings were that both infants and parents spontaneously used the pointing gesture in the same context across all cultures. Most infants between 10 and 14 months of age pointed with the index finger, and did so at about the same frequency, with no significant differences across cultures. Further, caregivers' and infants' pointing was overall correlated, thus revealing a social usage of the gesture from the beginning.

We have recently also begun testing infants' comprehension of the pointing gesture across cultures. Our previous findings of infants' comprehension of reference to occluded entities (Behne et al., submitted) are amenable to culture-specific interpretations, too. For example, it is possible that infants pass the hiding-finding game only because of extensive experience with such interaction frames at home, or because Western parents generally point a lot for their infants. We conducted the identical task of Behne and colleagues with traditionally living Yucatec Mayans in Mexico. Mayan infants have been reported to be socially less engaged, and parents hold the general belief that infants' communicative development is maturational, so that they engage in comparably less interactional formats with their infants (Gaskins, 1999, 2006; Salomo \& Liszkowski, 2010a, see next paragraph). We found that even the youngest Yucatec Mayan infants who we tested at 12 to 14 months of age performed virtually identically to their German counterparts as reported in Behne et al. (submitted). The study thus shows that the comprehension of prelinguistic pointing in referential terms is not a culture-specific phenomenon. Thus, both infants' production and comprehension skills of pointing are widespread and presumably universal skills of prelinguistic human communication.

Does the evidence that infants' prelinguistic communication is uniquely human, universal across cultures, and fully fledged already a year after birth require any further developmental account of its emergence? In a word, yes. Three-month-olds are perfectly able to extent the isolated index finger, as well as their arms, but why don't 3-month-olds point? The lack of continuity from the natural evolutionary line of development further forces us to develop an ontogenetic account of the emergence of pointing. In a recent study (Salomo \& Liszkowski, 2010a), we compared the natural occurrence of triadic joint interactional formats, in which infant and interlocutor(s) were engaged together with an object for at least 30 seconds, in the daily lives of three groups of 8- to 15-month-old infants in a Yucatec Mayan village, in a midsized Dutch city in the Netherlands, and in Shanghai, China. Using a scan-sampling method across 4 hours of home visits, we found statistically significant differences: Yucatec Mayan infants spent half as much time in triadic interactions as compared to Dutch infants, who again spent half as much time as Shanghai infants. This is the first study to reveal systematic quantitative differences across cultural settings in infants' triadic social interactions 
as they first emerge. In a further video recording of an hour of natural observations in the same groups of infants, we analyzed infants' and their partners' use of deictic gestures such as shows, offers, placings, and points. We found the same statistically significant pattern of cultural differences as before for infants' and their interlocutors' use of deictic gestures. Further, infants' gesture use correlated with their interlocutors' gesture use, as well as with the time spent in triadic interactions. The age of emergence of index-finger pointing within individual infants was significantly different across cultures.

The findings have two main implications. First, the social formats of preverbal triadic interaction, as well as basic deictic gestures, are likely used in all cultures by infants and their interactants, albeit with different frequencies. Second, the quantitative differences and the interrelations in the usage of these formats and gestures across cultures in both infants and interlocutors provide first evidence for a socially mediated ontogeny of prelinguistic communication skills. In particular, the fact that the individual ages of emergence of indexfinger pointing differed across cultures provides first evidence for the social mediation of this gesture. We can entail a scenario in which prelinguistic communication continues to be socially mediated after its emergence, leading all the way up to culturally specific forms of language socialization (Gaskins, 2006; Schieffelin \& Ochs, 1986) and relations between language and thought (e.g., Boroditsky, 2009). The current findings show that the beginnings of these foundational prelinguistic communication skills are already mediated by social processes in all cultures.

Further research along these lines needs to explore how the quantitative differences in infants' early prelinguistic communication relate to their emerging social cognitive abilities, thus following up and expanding on Vygotskian ideas of socioculturally mediated cognition.

\section{SUMMARY}

The unique and diverse complexities of human cognition emerge, by large, through social interactional processes and communication. However, social interaction and human communication themselves are based on deeper cognitive skills that are necessary to get communication off the ground. Prelinguistic infants are a test case to this hypothesis. The ontogenetic evidence demonstrates that infants indeed communicate in cognitively and motivationally rich ways, including communication about absent entities and an influence of unobservables such as others' knowledge, beliefs, and prior intentions. Infants are socially motivated to communicate spontaneously with others to align with or help them. Comparative research with nonhuman primates reveals fundamental differences in how chimpanzees and infants communicate in terms of referential and social intentions, demonstrating that 1-year-olds' prelinguistic communication is already a uniquely human form of communication. Cross-cultural research shows that core forms of infants' prelinguistic communication are indeed culturally universal, and that the specific pointing gesture is used universally in both production and comprehension. Further cross-cultural findings also reveal universal aspects in the input provided by caregivers. However, the quantitative cross-cultural differences in the input and its effect on infants' communication provide first evidence that socio-interactional practices indeed mediate infants' emerging communication skills. The naturally occurring frequency differences across cultural settings also provide the grounds for testing social origins accounts of social cognitive skills that are foundational to human communication. 


\section{REFERENCES}

Bates, E., Camaioni, L., \& Volterra, V. (1975). The acquisition of performatives prior to speech. MerrillPalmer Quarterly, 21, 205-226.

Bates, E. (1979). The emergence of symbols: Cognition and communication in infancy. New York: Academic Press.

Behne, T., Liszkowski, U., Carpenter, M., \& Tomasello, M. (2011). Twelve-month-olds' comprehension and production of informative pointing in a hiding-finding game. Manuscript submitted for publication.

Boroditsky, L. (2009). How does our language shape the way we think? In M. Brockman (Ed.), What's next? Dispatches on the future of science (pp. 116-129). New York: Vintage Press.

Bruner, J. S. (1981). The social context of language acquisition. Language \& Communication, 1(2-3), 155-178.

Bullinger, A. F., Zimmermann, F., Kaminski, J., \& Tomasello, M. (2010). Different social motives in the gestural communication of chimpanzees and human children. Developmental Science. doi:10.1111/ j.1467-7687.2010.00952.x

Butterworth, G. (2003). Pointing is the royal road to language for babies. In S. Kita (Ed.), Pointing: Where language, culture, and cognition meet (pp. 9-33). Mahwah, NJ: Lawrence Erlbaum Associates.

Call, J., \& Tomasello, M. (2007). The gestural communication of apes and monkeys. Mahwah, NJ: Lawrence Erlbaum Associates.

Carey, S. (2009). The origin of concepts. New York: Oxford University Press.

Carpendale, J. I. M., \& Lewis, C. (2004). Constructing an understanding of mind: The development of children's understanding of mind within social interaction. Behavioral and Brain Sciences, 27, 79-150.

Carpenter, M., Nagell, K., \& Tomasello, M. (1998). Social cognition, joint attention, and communicative competence from 9 to 15 months of age. Monographs of the Society of Research in Child Development, 63(4), Serial No. 176.

Delgado, B., Gómez, J. C., Sarriá, E. (2009). Private pointing and private speech: Developing parallelisms. In A. Winsler, C. Fernyhough, \& I. Montero (Eds.), Private speech, executive functioning, and the development of verbal self-regulation. New York: Cambridge University Press.

Desrochers, S., Morissette, P., \& Ricard, M. (1995). Two perspectives on pointing in infancy. In C. Moore \& P. J. Dunham (Eds.), Joint attention: Its origins and role in development (pp. 85-101). Hillsdale, NJ: Lawrence Erlbaum Associates.

Frank, M. C., Everett, D. L., Fedorenko, E., \& Gibson, E. (2008). Number as a cognitive technology: Evidence from Pirahã language and cognition. Cognition, 108, 819-824.

Gaskins, S. (1999). Children's daily lives in a Mayan village: A case study of culturally constructed roles and activities. In A. Goncu (Ed.), Children's engagement in the world: Sociocultural perspectives (pp. 25-81). Cambridge, United Kingdom: Cambridge University Press.

Gaskins, S. (2006). Cultural perspectives on infant-caregiver interaction. In N. J. Enfield \& S. C. Levinson (Eds.), Roots of human sociality: Culture, cognition and interaction (pp. 279-298). Oxford, United Kingdom: Berg.

Gliga, T., \& Csibra, G. (2009). One-year-old infants appreciate the referential nature of deictic gestures and words. Psychological Science, 20, 347-353.

Gomez, J. C., Sarria, E., \& Tamarit, J. (1993). The comparative study of early communication and theories of mind: Ontogeny, phylogeny, and pathology. In S. Baron-Cohen, H. Tager-Flusberg, \& D. J. Cohen (Eds.), Understanding other minds: Perspectives from autism (pp. 397-426). New York: Oxford University Press.

Grice, Paul (1957). Meaning. The Philosophical Review, 64, 377-388.

Haun, D. B. M., Rapold, C. J., Call, J., Janzen, G., \& Levinson, S. C. (2006). Cognitive cladistics and cultural override in Hominid spatial cognition. Proceedings of the National Academy of Sciences of the United States of America, 103(46), 17568-17573. 
Hockett, C. F. (1960). The origin of speech. Scientific American, 203, 88-96.

Knoblich, G., \& Sebanz, N. (2008). Evolving intentions for social interaction: From entrainment to joint action. Philosophical Transactions of the Royal Society B, 363, 2021-2031.

Knudsen, B., \& Liszkowski, U. (2010, March). 12- and 18-month-old infants warn others in anticipation of negative action outcomes. Poster presented at the International Conference on Infant Studies. Baltimore, USA.

Knudsen, B., \& Liszkowski, U. (2011). Infants helpfully correct other persons in anticipation of action mistakes. Manuscript submitted for publication.

Leavens, D. A., Russell, J. L., \& Hopkins, W. D. (2005). Intentionality as measured in the persistence and elaboration of communication by chimpanzees (pan troglodytes). Child Development, 76(1), 291-306.

Leung, E.H.L. \& Rheingold, H.L. (1981). Development of pointing as a social gesture. Developmental Psychology, 17(2), 215-220.

Levinson (2003). Space in language and cognition: Explorations in cognitive diversity. New York: Cambridge University Press.

Liebal, K., Carpenter, M., \& Tomasello, M. (2010). Infants' use of shared experience in declarative pointing. Infancy, 15, 545-556.

Liszkowski, U., Albrecht, K., Carpenter, M., \& Tomasello, M. (2008). Infants' visual and auditory communication when a partner is or is not visually attending. Infant Behavior and Development, 31(2), $157-167$

Liszkowski, U., Brown, P., Callaghan, T., Takada, A., \& de Vos, C. (2010, March). Cross-cultural comparisons of parent-infant pointing in 7 very different cultures. Poster presented at the International Conference on Infant Studies. Baltimore, USA.

Liszkowski, U., Carpenter, M., Henning, A., Striano, T., \& Tomasello, M. (2004). Twelve-month-olds point to share attention and interest. Developmental Science, 7(3), 297-307.

Liszkowski, U., Carpenter, M., Striano, T., \& Tomasello, M. (2006). Twelve- and 18-month-olds point to provide information for others. Journal of Cognition and Development, 7, 173-187.

Liszkowski, U., Carpenter, M., \& Tomasello, M. (2007a). Pointing out new news, old news, and absent referents at 12 months of age. Developmental Science, 10(2), F1-F7.

Liszkowski, U., Carpenter, M., \& Tomasello, M. (2007b). Reference and attitude in infant pointing. Journal of Child Language, 34(1), 1-20.

Liszkowski, U., Carpenter, M., \& Tomasello, M. (2008). Twelve-month-olds communicate helpfully and appropriately for knowledgeable and ignorant partners. Cognition, 108(3), 732-739.

Liszkowski, U., Schäfer, M., Carpenter, M., \& Tomasello, M. (2009). Prelinguistic infants, but not chimpanzees, communicate about absent entities. Psychological Science, 20, 654-660.

Liszkowski, U., \& Tomasello, M. (in press). Individual differences in social, cognitive, and morphological aspects of pointing at 12 months of age. Cognitive Development.

Luria, A. R. (1976). Cognitive development: Its cultural and social foundations. Cambridge, MA: Harvard University Press.

Moore, C., \& D'Entremont, B. (2001). Developmental changes in pointing as a function of attentional focus. Journal of Cognition \& Development, 2, 109-129.

Murphy, C. M. (1978). Pointing in the context of a shared activity. Child Development, 49(2), 371-380.

Povinelli, D. J., Bering, J. M., \& Giambrone, S. (2003). Chimpanzees' “pointing”: Another error of the argument by analogy? In S. Kita (Ed.), Pointing: Where language, culture, and cognition meet (pp. 35-68). Mahwah, NJ: Lawrence Erlbaum Associates.

Quine, W. (1960). Word and object. Cambridge, MA: Harvard University Press.

Richerson, P. R., \& Boyd, R. (2005). Not by genes alone. How culture transformed human evolution. Chicago: Chicago University Press.

Salomo, D., \& Liszkowski, U. (2010a, March). Caretaker-infant social interaction and gestures across cultures. Poster presented at the International Conference on Infant Studies, Baltimore. 
Salomo, D., \& Liszkowski, U. (2010b, March). Yucatec-Mayan infants comprehend the communicative intent behind pointing gestures. Poster presented at the International Conference on Infant Studies. Baltimore, USA.

Schieffelin, B., \& Ochs, E. (Eds.). (1986). Language socialization across cultures. New York: Cambridge University Press.

Searle, J. R. (1969). Speech acts. Cambridge: Cambridge University Press.

Spelke, E. S. (1994). Initial knowledge: Six suggestions. Cognition, 50, 431-445.

Sperber, D., \& Wilson, D. (1995). Relevance: Communication and cognition (2nd ed.). Oxford, United Kingdom: Blackwell.

Tinbergen, N. (1951). The study of instinct. New York: Oxford University Press.

Tomasello, M. (1999). The cultural origins of human cognition. Cambridge, MA: Harvard University Press.

Tomasello, M. (2006). Why don't apes point? In N. Enfield \& S. Levinson (Eds.), Roots of human sociality: Culture, cognition, and interaction. Oxford, United Kingdom: Berg.

Tomasello, M. (2008). Origins of human communication. Cambridge, MA: MIT Press.

Tomasello, M., Carpenter, M., Call, J., Behne, T., \& Moll, H. (2005). Understanding and sharing intentions: The origins of cultural cognition. Behavioral and Brain Sciences, 28, 675-691.

Tomasello, M., Carpenter, M., \& Liszkowski, U. (2007). A new look at infant pointing. Child Development, 78, 705-722.

Vygotsky, L. S. (1962). Thought and language. Cambridge, MA: MIT Press.

Vygotsky, L. S. (1978). Mind in society: The development of higher psychological processes. Cambridge, MA: Harvard University Press.

Wilkins, D. (2003). Why pointing with the index finger is not a universal. In Sotaro Kita (Ed.) Pointing: Where language, culture, and cognition meet (pp. 171-215). Mahwah, NJ: Lawrence Erlbaum Associates.

Acknowledgements. I am thankful to Susan Schmidt and two other reviewers for helpful comments on an earlier draft.

Correspondence regarding this article should be directed to Ulf Liszkowski, Max Planck Institute for Psycholinguistics, Wundtlaan 1, 6525 XD Nijmegen The Netherlands. E-mail: ulf.liszkowski@mpi.nl 